in cleanliness, visualization and positive rates. But groups of 30 to 60 minutes got the least supplement of water during MCE, and it may be the best interval time between gastric preparation and MCE.

\section{IDDF2020-ABS-0044 TO EVALUATE THE FEASIBILITY AND EFFICACY OF NON-RADIOLOGICAL TEST TO DETECT POST OPERATIVE LEAK AFTER BARIATRIC SURGERY}

Nikhil Gupta*, Shubham Goel, Arun K Gupta, ASN Rao. ABVIMS Dr RML Hospital, India

\subsection{6/gutjnl-2020-IDDF.52}

Background Gastrograffin study under fluoroscopic guidance is a norm after sleeve gastrectomy in most of the bariatric centres to detect the post operative leak. Shifting the patient to fluoroscopic room on post operative day one and radiation exposure are major concerns associated with this protocol. A lot of studies are available to prove the efficacy of bed side oral methylene blue test to detect anastomotic leak after esophagojejunal anastomosis. We used the same principle in our bariatric patients.

Methods This prospective, interventional study was conducted on 23 patients undergoing sleeve gastrectomy for morbid obesity at our institution.

Results All patients were haemodynamically stable on post operative day-1. There was no evidence of methylene blue in the drain in any of the patients. All patients were started oral liquids after the test.

Conclusions Routine gastrograffin study under fluoroscopic guidance in the post operative period to detect leak following sleeve gastrectomy is a cumbersome procedure and can be avoided. Oral bed side Methylene blue test is a good alternative.

\section{IDDF2020-ABS-0045 CLINICAL SIGNIFICANCE OF INTRA- ABDOMINAL PRESSURE MEASUREMENT IN PATIENTS WITH ACUTE ABDOMEN REQUIRING EXPLORATORY LAPAROTOMY}

${ }^{1}$ Binita Goswami*, ${ }^{2}$ Sumit Pathania, ${ }^{2}$ Arun K Gupta, ${ }^{2}$ Nikhil Gupta. ${ }^{1}$ Maulana Azad Medical College, Delhi, India; ${ }^{2} A B V I M S$ Dr RML Hospital, India

\subsection{6/gutjnl-2020-IDDF.53}

Background Intra-abdominal Hypertension (IAH) has been identified as a significant risk factor for morbidity and mortality. Our study was done to monitor intra-abdominal pressure (IAP) in patients with acute abdomen requiring exploratory laparotomy and to determine its effect on multiple organs by measuring SOFA score and its effect on the outcome of patients.

Methods It was an observational study in which IAP was measured pre and post-operatively by inserting a catheter in the urinary bladder using manometer 6 hourly in patients of acute abdomen requiring exploratory laparotomy.

Results Of 60 patients 23 (38\%) had IAP (mean) and 24 (40\%) had IAP (max) more than $12 \mathrm{mmHg}$. Patients with intestinal obstruction and intestinal perforation with peritonitis had an incidence of raised IAP. There was an increase in hospital stay in patients with IAH, and this was statistically significant $(p<0.05)$. There was positive correlation coefficient relationship between IAP (mean) and (max) with hospital stay $r \operatorname{IAP}(\max )=0.4757$ and $\mathrm{r} \operatorname{IAP}(\max )=0.4893$. Elevated IAP affects all organs, but cardiovascular, renal and respiratory systems were more prone to failure, and there was a positive correlation between IAP and organ failure.

Conclusions IAP should be routinely measured in patients of acute abdomen requiring exploratory laparotomy. Patients with raised IAP preoperatively should be taken up for emergency surgery as soon as possible for a better outcome before deleterious effects of raised IAP on various organ system set in.

\section{IDDF2020-ABS-0048 STUDY ON THE CHANGES OF COAGULATION INDEXES IN PATIENTS WITH CROHN'S DISEASE}

Yi Yu*. Department of Critical Care Medicine, The Second Affiliated Hospital of Guangzhou University of Chinese Medicine, China

\subsection{6/gutjnl-2020-IDDF.54}

Background Thromboembolism is a life-threatening complication of Crohn's disease(CD), CD patients were four times more likely to develop thromboembolism than healthy people, its relative risk increased to 15 times during the active period of disease. To investigate the association between the changes of coagulation indexes in patients with CD.

Methods The clinical data of $78 \mathrm{CD}$ patients who diagnosed for the first time (CD group) and 75 health checkup (control group) from June 2010 to July 2018 in the MIMIC-III database were analyzed retrospectively. The coagulation indexes of the two groups and those of patients with different activity in the CD group were compared, the correlation between coagulation indexes and Crohn's disease activity index (CDAI) scores of Crohn's disease were analyzed.

Results The levels of fibrinogen, platelet count and PT in the CD group were significantly higher than those in the healthy control group $(\mathrm{P}<0.05)$. The levels of fibrinogen, FDP, and APTT in patients with remission stage were significantly lower than those in patients with moderate activity stage $(\mathrm{P}<0.05)$. Pearson linear correlation analysis showed that fibrinogen, platelet count, FDP and APTT were positively correlated with CDAI $(\mathrm{P}<0.05)$.

Conclusions The coagulation indexes of CD patients are significantly higher than those of normal people, and fibrinogen, platelet count, FDP and APTT are closely related to the activity of Crohn's disease.

\section{IDDF2020-ABS-0049 APPLICATION OF NUTRITIONAL RISK SCREENING AND BMI IN NUTRITIONAL ASSESSMENT OF PATIENTS WITH CROHN'S DISEASE}

Yi Yu*. Department of Critical Care Medicine, The Second Affiliated Hospital of Guangzhou University of Chinese Medicine, China

\subsection{6/gutjnl-2020-IDDF.55}

Background To investigate the association between the nutritional risk screening (NRS-2002) and body mass index (BMI) were used to assess the nutritional status of patients with Crohn's disease.

Methods 134 patients with Crohn's disease admitted to the First Affiliated Hospital of Sun Yat-sen University from June 
2013 to May 2018 were selected as subjects. NRS-2002 and BMI were used to assess the nutritional status of the entire group of patients, comparing the nutritional status of patients with Crohn's disease with intestinal fistula and non-intestinal fistula.

Results 66 patients with Crohn's disease had an NRS $\geq 3$ points in 134 cases; the incidence rate was $49.25 \%(66 / 134)$; $\mathrm{BMI}<18.5 \mathrm{~kg} / \mathrm{m}^{2}$ in 39 cases, the incidence rate was $29.10 \%$ (39/134). 19 patients with NRS $\geq 3$ points with non-intestinal fistula, the incidence rate was $28.78 \%(19 / 66)$; 9 patients with BMI $<18.5 \mathrm{~kg} / \mathrm{m}^{2}$ with intestinal fistula, the incidence rate was $23.07 \%$ (9/39). In patients with intestinal fistula, 47 patients had an NRS $\geq 3$, the incidence rate was $71.21 \%(47 /$ 66 ), and 30 patients with BMI $<18.5 \mathrm{~kg} / \mathrm{m}^{2}$, the incidence rate was $76.92 \%(30 / 39)$. The incidence of nutritional risk and malnutrition in patients with intestinal fistula was higher than that in patients with non-intestinal fistula (nutrition risk incidence: $71.21 \%$ vs $28.78 \%$; $<<0.05$; incidence of malnutrition: $76.92 \%$ vs $23.07 \%$; $<0.05$ ).

Conclusions Patients with Crohn's disease have a high incidence of nutritional risk and malnutrition. Patients with Crohn's disease and intestinal fistula have higher nutritional risk and malnutrition rates than patients with nonintestinal fistula. Therefore, nutritional support for patients with Crohn's disease during the perioperative period should be strengthened, especially in patients with intestinal fistula.

\section{IDDF2020-ABS-0054 CLINICAL STUDY ON THE CHANGES OF SERUM HCY AND PC IN PATIENTS WITH CROHN'S DISEASE}

Yi Yu*. Department of Critical Care Medicine, the Second Affiliated Hospital of Guangzhou University of Chinese Medicine, China

\subsection{6/gutjnl-2020-IDDF.56}

Background To investigate the changes of Hcy and PC in CD patients, and further explore the related factors of the prethrombotic state of CD patients.

Methods We collected clinical data of 65 patients with CD in our hospital from January 2012 to June 2016. 67 health examiners as controls. Fasting venous blood was collected in the morning. The serum Hcy and PC in CD patients were detected by ELISA method. The results of the test were compared with those in the control group, and prethrombotic state correlation analysis was also performed.

Results Compared with the control group, the serum Hcy in $\mathrm{CD}$ patients increased significantly $(6.78 \pm 2.17 \mathrm{ng} / \mathrm{ml}$ vs. $12.34 \pm 6.11 \mathrm{ng} / \mathrm{ml})$, and the difference was statistically significant $(\mathrm{P}<0.05)$, the serum $\mathrm{PC}$ in $\mathrm{CD}$ patients slight increased $(7.44 \pm 3.15 \mathrm{ng} / \mathrm{ml}$ vs $7.23 \pm 3.58 \mathrm{ng} / \mathrm{ml})$, while the difference was not statistically significant $(P>0.05)$. The correlation analysis showed that Hcy and PC levels in CD patients were positive correlate with prethrombotic state $(r=0.317$, $\mathrm{P}<0.05)$.

Conclusions The serum Hcy in CD patients was significantly higher than those in the control group. The correlation analysis showed that Hcy and PC levels in CD patients were positive correlate with prethrombotic state, these may due to the decrease of antithrombotic factors, and finally having a potential risk of thrombosis in $\mathrm{CD}$ patients.

\section{IDDF2020-ABS-0055 THE LONG-TERM OUTCOME OF ENDOSCOPIC RADIO INCISION WITH BALLOON DILATION OR WITH ESOPHAGEAL STENT PLACEMENT IN LONG-SEGMENT BENIGN ESOPHAGEAL STRICTURES}

Jui-yen Chen*, Jia-chuan Wu, Xiao-dong Chen, Li-fang Ye, Xiao-qiao Yang, Biao Liang Guangdong Second Provincial General Hospital, China

\subsection{6/gutjnl-2020-IDDF.57}

Background Endoscopic radio incision (ERI) is a novel treatment for benign esophageal strictures, especially for refractory stenoses. ERI provides a rapid improvement patency and exciting result in the short term. However, re-incision and/or dilation are required in some patients with long-segment strictures, and the long-term outcome of ERI is still controversial. The aim of this study was to evaluate the long-term efficacy of ERI with balloon dilation (BD) and ERI with esophageal stent placement (ESP) in long-segment benign esophageal strictures.

Methods This study was a randomized prospective trial. 37 patients with $1.5-5 \mathrm{~cm}$ length benign esophageal strictures from July 2015 to December 2018 were enrolled (the median age was 67 years old, range 49-74; male: female 25:12) and followed up for 12 months. All patients were randomly assigned to two groups: the ERI with balloon dilation group (ERI+BD, $\mathrm{n}=18)$ and ERI with esophageal stent placement group $(\mathrm{ERI}+\mathrm{ESP}, \mathrm{n}=19)$. The clinical data were recorded, such as the diameter of stenoses, length of strictures, dysphagia scores before and after the procedure, complications.

Results No severe adverse events were observed in either group. All patients in both groups were able to eat solid food within 1 week after the treatment. There was no significant difference between the ERI+BD group and the ERI+ESP group in dysphagia scores $(1.3 \pm 0.5$ vs $1.1 \pm 0.4, P>0.05)$ within 4 weeks after the procedure. In a 1-year follow-up, the relief rate of dysphagia symptom in the ERI+ESP group was higher than the ERI+BD group $(84.2 \%$ vs $44.4 \%, P=$ 0.029). Comparing to the ERI+BD group, the majority of patients in the ERI+ESP group could maintain lumen patency at 12 months $(78.9 \%$ vs $38.9 \%, P=0.032)$.

Conclusions Endoscopic radio incision (ERI) with esophageal stent placement (ESP) is the effective treatment for long-segment benign esophageal stricture, and provides a favorable result in the long-term.

\section{IDDF2020-ABS-0068 3D MODEL RECONSTRUCTION OF THE WHOLE STOMACH FROM STANDARD ENDOSCOPE VIDEO}

${ }^{1}$ Sho Suzuki*, ${ }^{2}$ Kenji Miki, ${ }^{1}$ Takuji Gotoda, ${ }^{3}$ Aji Resindra Widya, ${ }^{3}$ Yusuke Monno, ${ }^{3}$ Masatoshi Okutomi. 'Division of Gastroenterology and Hepatology, Department of Medicine, Nihon University School of Medicine, Japan; ${ }^{2}$ Department of Internal Medicine, Tsujinaka Hospital Kashiwanoha, Japan; ${ }^{3}$ Department of Systems and Control Engineering, School of Engineering, Tokyo Institute of Technology, Japan

\subsection{6/gutjnl-2020-IDDF.58}

Background Endoscopy is a common clinical practice to evaluate gastrointestinal diseases. Although endoscopy assesses gastrointestinal mucosal surface, it cannot evaluate 